\title{
PRODUÇÃO CIENTÍFICA NA ÁREA DA SAÚDE SOBRE SÍFILIS GESTACIONAL: REVISÃO NARRATIVA
}

SCIENTIFIC WORKS IN HEALTH SCIENCES ON GESTATIONAL SYPHILIS: A NARRATIVE REVIEW

PRODUCCIÓN CIENTÍFICA EN EL ÁREA DE LA SALUd ACERCA DE SÍFILIS GESTACIONAL: UNA REVISIÓN NARRATIVA

Natália da Silva Gomes ${ }^{1}$

Lisie Alende Prates 2

Rhayanna de Vargas Perez ${ }^{3}$

Camila Xavier Fialho 4

Mari Luci Chalme da Silva ${ }^{5}$

Pâmela da Rosa Gonzalez ${ }^{6}$

Palavras-chave:

Sifilis; Sorodiagnóstico da Sifilis; Gestantes; Gravidez.

Keywords:

Syphilis; Syphilis Serodiagnosis; Pregnant Women; Pregnancy.

Palabras clave: Sífilis; Serodiagnóstico de la Sífilis; Mujeres Embarazadas; Embarazo.

Submetido: $17 / 01 / 2020$

Aprovado: 12/05/2020

Autor(a) para Correspondência: Lisie Alende Prates

Rua Dr. Maia, 2160, apto. 302 Bela Vista - Uruguaiana (RS) CEP: 97501-768

E-mail: lisiealende@hotmail. com

\section{RESUMO}

Este estudo teve por objetivo identificar as evidências cientificas disponíveis na literatura da área da saúde sobre sifilis gestacional. A revisão narrativa foi desenvolvida na base de dados Literatura Latino-Americana e do Caribe em Ciências da Saúde (LILACS) e na Base de Dados de Enfermagem (BDENF), com os descritores "sífilis", "sorodiagnóstico de sifilis", "gestantes" e "gravidez". Dentre os 34 artigos analisados, verificou-se que tratavam de assistência, prevalência, prevenção, diagnóstico e tratamento da sifilis gestacional. Os achados permitem identificar um déficit na qualidade da assistência prestada à gestante portadora de sífilis. Identificou-se a importância da adesão ao tratamento e da inserção dos parceiros sexuais nesse seguimento, tendo em vista sua relevância para a prevenção das infecções sexualmente transmissíveis (IST). Ao mesmo tempo, esta revisão permitiu apontar um caminho para o desenvolvimento de pesquisas e ações assistenciais direcionadas à prevenção da sifilis no período gestacional.

1. Acadêmica de Enfermagem na Universidade Federal do Pampa (Unipampa). Membro do Grupo de Pesquisas e Estudos na Saúde da Mulher (GRUPESM). Bolsista do Programa de Educação Tutorial - Práticas Integradas em Saúde Coletiva (PET-PISC).E-mail: nataliasilvag_@hotmail.com 0RCID: http://orcid.org/0000-0002-6952-7172

2. Enfermeira. Doutora em Enfermagem. Professora na Unipampa. Líder do GRUPESM. E-mail: lisiealende@hotmail. com- ORCID: http://orcid.org/0000-0002-5151-0292

3. Acadêmica de Enfermagem na Unipampa. Membro do GRUPESM. E-mail: rhayannaperez@hotmail.com 0RCID: http://orcid.org/0000-0003-2289-8141

4. Acadêmica de Enfermagem na Unipampa. Membro do GRUPESM. E-mail: camila.xf@hotmail.com 0RCID: http:// orcid.org/0000-0003-4093-6869

5. Acadêmica de Enfermagem na Unipampa. Membro do GRUPESM. E-mail: mari.chalme02@gmail.com 0RCID: http://orcid.org/0000-0002-7632-0751

6. Acadêmica de Enfermagem na Unipampa. Membro do GRUPESM. E-mail: pame.gonz@hootmail.com ORCID: http://orcid.org/0000-0003-1961-4398

Certificação de redação científica: E.L.Freire Editora. Edição de texto: Evandro L. Freire. Preparação de original: Dida Bessana. 


\section{ABSTRACT}

This study aimed to identify the scientific evidence available in the literature in health sciences concerning gestational syphilis. The narrative review was conducted in the database Literature in the Health Sciences in Latin America and the Caribbean (LILACS) and in the Nursing Database (BDENF), using the descriptors "syphilis," "syphilis serodiagnosis," "pregnant women," and "pregnancy." Among the 34 articles analyzed, it was found that they addressed gestational syphilis's care, prevalence, prevention, diagnosis, and treatment. The findings allow us to identify a poor quality of care provided to pregnant women with syphilis. The importance both of treatment adherence and sexual partners' participation in follow-up care was identified, considering their relevance to prevent sexually transmitted infections (STIS). At the same time, this review allowed us to point out a path for further research and new clinical actions aimed at syphilis prevention within the gestational period.

\section{RESUMEN}

Este estudio tuvo como objetivo identificar la evidencia científica disponible en la literatura del área de la salud acerca de la sifilis gestacional. La revisión narrativa se desarrolló en la base de datos Literatura Latinoamericana y del Caribe en Ciencias de la Salud (LILACS) y en la Base de Datos de la Enfermaría (BDENF), con los descriptores "sífilis", "serodiagnóstico de sífilis", "mujeres embarazadas" y "embarazo". Entre los 34 artículos analizados, se encontró que se ocupaban de la asistencia, la prevalencia, la prevención, el diagnóstico y el tratamiento de la sifilis gestacional. Los hallazgos permiten identificar un déficit en la calidad de la atención brindada a las mujeres embarazadas con sifilis. Se identificó la importancia de la adherencia al tratamiento y la inserción de las parejas sexuales en este seguimiento, en vista de su relevancia para la prevención de infecciones de transmisión sexual (ITS). Al mismo tiempo, esta revisión permitió señalar un camino para el desarrollo de nuevas investigaciones y acciones de atención dirigidas a la prevención de la sífilis durante el periodo gestacional.

\section{INTRODUÇÃ 0}

A sífilis é uma doença infecciosa sistêmica, causada pela espiroqueta da bactéria gram-negativa Treponema pallidum. Apresenta evolução crônica e pode ser transmitida por via sexual ou vertical, ocorrendo, ainda, de forma adquirida e congênita. A forma adquirida se subdivide em recente e tardia, dependendo do tempo de infecção e do grau de infectividade, e a forma congênita é transmitida por via transplacentária ${ }^{1}$.

A sifilis recente se manifesta em menos de 2 anos após o contato. É subdividida em 3 estágios: primário, secundário e latente recente. 0 tempo de incubação do estágio primário é, em média, de 21 dias depois do contato e tem como sinal clínico um nódulo indolor único no local do contato, que ulcera rapidamente, formando o cancro duro. 0 segundo estágio ocorre de 6 semanas a 6 meses, depois do contato. Nessa fase, são comuns sinais e sintomas sistêmicos, erupções cutâneas e lesões eritemato-escamosas palmo-plantares. Por fim, o estágio latente recente ocorre nos primeiros 2 anos de infecção, sem que se observe nenhum sintoma clínico, apenas reatividade nos testes imunológicos, podendo ser facilmente confundida com outra doença².
$\mathrm{Na}$ sífilis tardia, os sintomas se revelam depois de mais de 2 anos desde o contato bacteriano, e é subdividida em 2 estágios: latente tardio e terciário. 0 estágio latente tardio se mantém assintomático e no terciário pode ocorrer o acometimento do sistema nervoso e cardiovascular ${ }^{2}$.

A sifilis congênita é o resultado da infecção por via transplacentária do Treponema pallidum. Ocorre em gestante infectada, tratada inadequadamente ou não tratada. A infecção do recém-nascido pode ser assintomática ou sintomática, sendo classificada em 2 estágios: precoce, quando é diagnosticada até 2 anos de vida e tardia, depois desse período ${ }^{3}$.

A sifilis gestacional, apesar de apresentar diagnóstico simples e tratamento eficaz, permanece com prevalência alarmante, sobretudo em países subdesenvolvidos ou em desenvolvimento ${ }^{1}$. Suas manifestações clínicas se assemelham às encontradas na sifilis adquirida.

No Estado do Rio Grande do Sul, segundo o Sistema de Informática do Sistema Único de Saúde (DataSUS), em 2017, foram notificados 3.456 casos de sífilis gestacional, cifra que não mudou muito em 2018 (3.723 casos notificados) ${ }^{4}$.

Diante do exposto, este estudo teve por objetivo identificar as evidências científicas disponíveis na literatura da área da saúde sobre sífilis gestacional. 


\section{METODOLOGIA}

Trata-se de uma revisão narrativa da literatura, método que envolve temáticas amplas. Nesse tipo de revisão, a seleção dos estudos e a interpretação das informações podem estar sujeitas à subjetividade dos autores ${ }^{5}$.

A pergunta de pesquisa adotada foi: Qual o atual status da produção científica na área da saúde sobre sífilis gestacional?

Em setembro de 2018, a busca dos artigos foi feita na base de dados Literatura Latino-Americana e do Caribe em Ciências da Saúde (LILACS) e na Base de Dados de Enfermagem (BDENF). Como descritores, consultados na página dos Descritores em Ciências da Saúde (DeCS), adotamos "sífilis", "sorodiagnóstico da sífilis", "gestantes" e "gravidez".

Os critérios de inclusão englobaram artigos: originais; disponíveis gratuitamente on-line; publicados em português; e publicados a partir do ano 2000. Neste ano foi criado o Programa de Humanização no Pré-Natal e Nascimento (PHPN), o qual evidenciou questões ligadas à qualidade da assistência pré-natal e à taxa de incidência de sífilis congênita no Brasil ${ }^{6}$. Pode-se considerar o PHPN um marco em relação à visibilidade da prevenção da doença no Brasil. Logo, adotou-se esse programa como referência temporal para a busca.

Já o critério de exclusão adotado foi: Publicações que não se enquadraram na área da saúde.

Depois da definição dos descritores, a busca nas bases de dados LILACS e BDENF ocorreu da seguinte maneira: "SÍFILIS" “OR" “sorodiagnóstico da SÍfILIS" [Descritor de assunto] “AND" (“GESTANTES) “OR" “GRAVIDEZ" [Descritor de assunto] "AND" “PORTUGUES" [Idioma].

Foram identificados 99 artigos (90 na LILACS e 9 na BDENF). Destes, 34 artigos foram selecionados para análise, sendo os demais excluídos com base nos seguintes critérios (Figura 1): Não é artigo original (26); foi publicado em outro idioma (7); não dispõe de resumo completo (30); e foi publicado antes de 2000 (2).

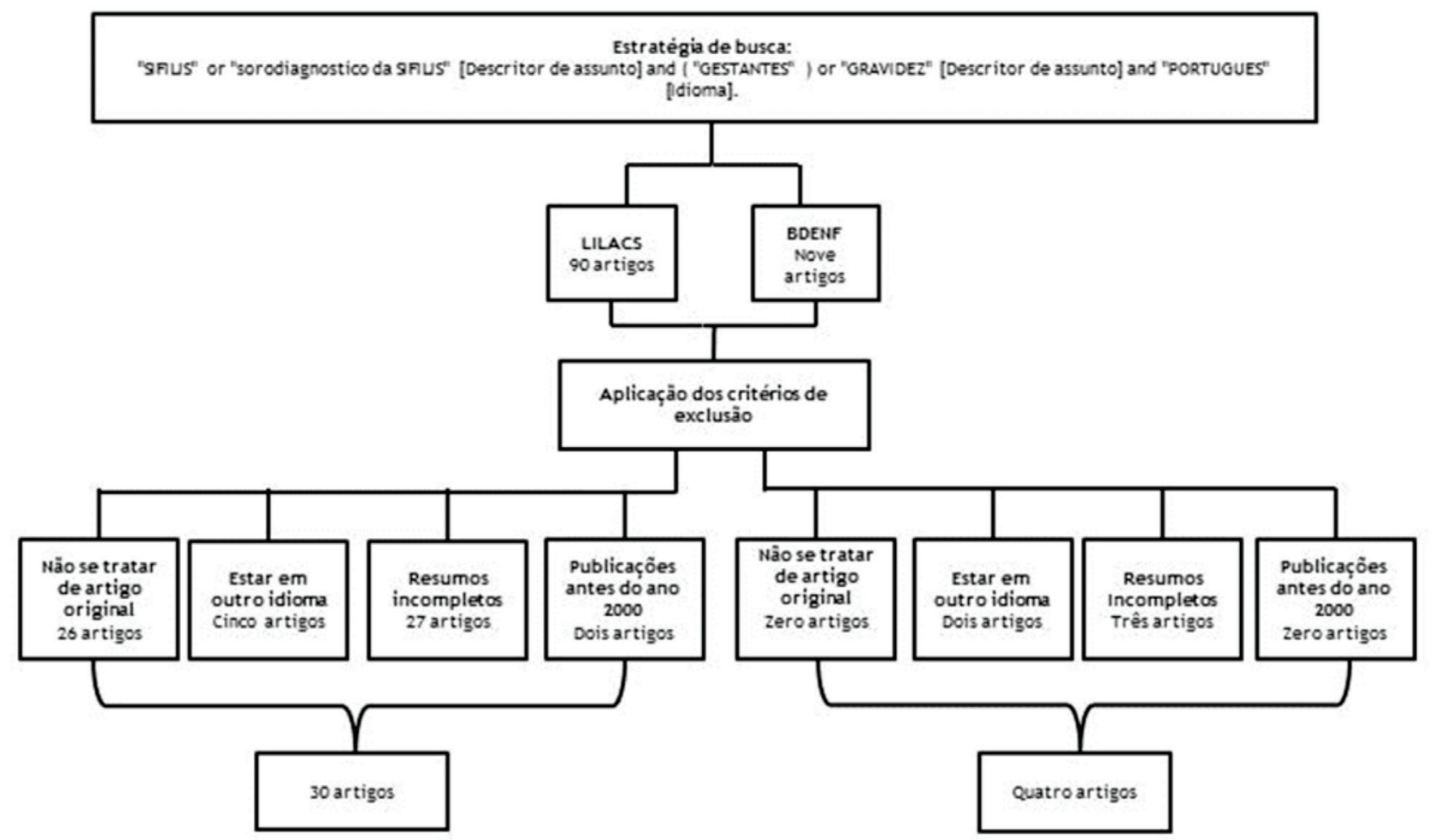

Figura 1. Fluxograma dos artigos selecionados.

Fonte: Elaborada pelos autores.

Em seguida, a produção científica na área da saúde sobre sífilis gestacional foi dividida em 5 tópicos para análise das evidências: a) assistência; b) prevalência; c) prevenção; d) diagnóstico; e e) tratamento. 


\section{RESULTADOS}

As publicações selecionadas nesta revisão foram desenvolvidas por diferentes profissionais da saúde: $24(70,58 \%)$ por médicos; $6(17,64 \%)$ por enfermeiros; $2(5,88 \%)$ por profissionais ligados às ciências biológicas; e $1(2,94 \%)$ por acadêmico de enfermagem e farmacêutico. Quanto ao ano de publicação, foram identificadas: 1 produção $(2,94 \%)$ nos anos 2000, 2006, 2014 e 2017; 2 (5,88\%) em $2001,2004,2007$ e 2011; 3 (8,82\%) em 2008, 2009, 2010 e 2013 ; $6(17,64 \%)$ em 2012 ; e $4(11,76 \%)$ em 2016.

Acerca do cenário de estudo, os hospitais predominaram (19 [55,88\%] dos achados); mas também foram encontrados 11 estudos (32,35\%) na atenção primária à saúde (APS); 1 (2,94\%) em casa de parto; $2(5,88 \%)$ em centro de testagem e aconselhamento (CTA); e 2 (5,88\%) em busca no DataSUS. As pesquisas foram conduzidas com diferentes participantes, dentre os quais: 29 $(85,29 \%)$ gestantes; $3(8,82 \%)$ parturientes; 3 $(8,82 \%)$ com profissionais da saúde; e 1 (2,94\%) com gerentes setoriais e gestores. Em relação ao método de estudo, predominaram os descritivos (14 $[41,17 \%])$ e os transversais $(10[29,41 \%])$. Também foram identificados $3(8,82 \%)$ estudos transversais e descritivos e 1 (2,94\%) estudo longitudinal e retrospectivo.

Em seguida, apresentam-se os tópicos em que foram divididas as evidências científicas disponíveis na área da saúde sobre sífilis gestacional: a) assistência; b) prevalência; c) prevenção; d) diagnóstico; e e) tratamento.

No âmbito assistencial, os resultados foram diferentes para cada público. Para as adolescentes, - nível de escolaridade pode ser considerado um fator determinante para as infecções sexualmente transmissiveis (IST) ${ }^{7}$. Jovens e adolescentes com baixa escolaridade são mais suscetíveis às IST, pois pouco se preocupam com os aspectos preventivos, não sendo uma falha assistencial de informação, mas um aspecto comportamental inerente à faixa etária ${ }^{8}$.

As gestantes adultas não souberam informar a importância dos exames solicitados no pré-natal, demonstrando que os profissionais da saúde não as orientaram sobre os exames antes de solicitá-los. Em um estudo que analisou profissionais da saúde, gestantes e prontuários, houve discrepância entre as informações. As gestantes revelaram restrição de informações prestadas pelos profissionais da saúde

\section{As gestantes adultas não souberam informar a importância dos exames solicitados no pré-natal...}

sobre o exame VDRL (sigla em inglês do termo venereal disease research laboratory) e o teste rápido; já os profissionais da saúde afirmaram que compartilham seu conhecimento sobre os exames diagnósticos e o tratamento na assistência pré-natal9-10. Outra situação importante a considerar diz respeito à falta de registro sobre o tratamento do parceiro sexual ${ }^{11}$.

Quanto à sífilis congênita, a trajetória assistencial das gestantes evidenciou início tardio do pré-natal e ausência de diagnóstico na gravidez ${ }^{12}$. Há uma lacuna na assistência a essas gestantes, que pode estar associada a dificuldades apresentadas pelos profissionais da saúde no manejo clínico da sífilis, resultando em pré-natais abaixo dos níveis preconizados pelo Ministério da Saúde (MS), diagnóstico tardio e falta de adesão ao tratamento dessas gestantes ${ }^{13}$.

Os resultados sobre a prevalência de sífilis em gestantes demonstraram que gestantes com idade entre 20 e 34 anos têm maior risco de exposição à infecção, que ocorre na maioria das gestações ${ }^{14}$. Observou-se correlação estatisticamente significativa entre VDRL positivo e o grau de instrução materno, assim como o número de consultas pré-natal. Quanto maior a instrução materna e o número de consultas durante o pré-natal, menor a prevalência de VDRL positivo ${ }^{14-15}$.

Quando comparada a outros países, em 2004, a Colômbia apresentou prevalência 2 vezes maior de sífilis do que o Brasil e o Peru ${ }^{16}$. Mesmo esse número sendo baixo, pode ter relação com as subnotificações, pois os resultados demonstraram que a vigilância epidemiológica raramente faz busca ativa para sífilis congênita. Mesmo assim, a transmissão vertical ainda é prevalente se comparada a outras IST $^{17}$.

Os resultados para prevenção mostraram que maternidades com o Projeto Nascer implantado não contavam com ações de aconselhamento e autorização para a realização dos testes rápidos da sífilis, sendo esta uma conduta desencadeadora de todas as estratégias de precaução da transmissão vertical ${ }^{18-19}$. Sobre os óbitos fetais antes da hospitalização 
materna, a ausência do exame VDRL, no pré-natal, foi identificada como a principal consequência ${ }^{20}$.

Ficou evidente nos resultados encontrados que a identificação de barreiras para a adoção de protocolos assistenciais se mostra fundamental para a formulação de estratégias de intervenção e prevenção, além do uso de outras abordagens de educação continuada dos profissionais da saúde ${ }^{21}$. Pode-se pensar na redução da sífilis gestacional com prevenção por meio de estratégias vinculadas à assistência e à total inclusão do parceiro no prénatal ${ }^{22-23}$.

As evidências científicas sobre os diagnósticos destacam a necessidade do desenvolvimento de testes treponêmicos e não treponêmicos para a sifilis, com o intuito de acompanhar as quedas de titulação de pacientes em tratamento. Em um dos estudos, evidenciou-se que mesmo o exame VDRL sendo muito adotado para diagnóstico, ele indica resultados falso-positivos e falso-negativos com frequência - o que, muitas vezes, trata-se de um seguimento do efeito prozona ${ }^{24-25}$.

Depois do diagnóstico da gestante, os resultados evidenciaram que o tratamento com penicilina precisa ser imediato e é o mais recomendado, pois outros medicamentos não apresentam eficácia comprovada no que se refere à sífilis congênita ${ }^{26}$. Quanto aos parceiros sexuais, um estudo mostrou que não fazem o tratamento adequado por recusa, por não apresentarem sintomas clínicos, por não acreditarem no tratamento e/ou por terem medo da administração do medicamento ${ }^{27}$.

No cuidado ao recém-nascido exposto à sífilis, o tratamento não ocorre em seguimento, muitas vezes pela distância entre a residência e o serviço de saúde ou nos casos em que puérperas têm mais de 3 filhos e ausência de coinfecção nas gestações anteriores. Já as mães adolescentes aderem mais ao tratamento de seus filhos, visto que ainda não estão inseridas no mercado de trabalho e recebem ajuda de outros familiares ${ }^{28}$.

\section{DISCUSSÃO}

Neste estudo, identificou-se que algumas fragilidades encontradas na assistência influenciam significativamente o quadro clínico de gestantes com sífilis. 0 diagnóstico tardio durante o pré-natal pode ser considerado uma das maiores fragilidades, ao que se soma o tratamento inadequado das gestantes e de seus parceiros. As consequências dessas diversas falhas contribuem para o aumento da prevalência da doença e permitem levantar a hipótese de que a prevenção dessas gestantes pode não estar sendo feita corretamente.

As desigualdades sociais em saúde parecem apoiar a hipótese de que a sifilis gestacional se relaciona ao baixo nível socioeconômico e ao atendimento pré-natal inadequado. 0 atendimento inadequado, por exemplo, contribui para a persistência da transmissão vertical e expõe as fragilidades da assistência, não só quanto ao acesso, mas também em relação à oportunidade de rastreamento e tratamento das gestantes e seus parceiros ${ }^{29}$.

Os parceiros sexuais geralmente não comparecem ao serviço de saúde ou, quando presentes, recusamse a fazer exames e/ou submeter-se a tratamento. Um estudo revelou que, depois da comunicação do diagnóstico de sífilis gestacional, habitualmente os parceiros procuram o serviço de saúde para que seja administrada a primeira dose de penicilina, mas não voltam para dar seguimento ao tratamento ${ }^{30}$.

Segundo a Organização Mundial da Saúde (OMS), o número adequado de consultas pré-natal precisa ser igual ou superior a seis ${ }^{31}$. Durante esse acompanhamento, o teste rápido de sífilis e o exame VDRL precisam ser solicitados e feitos no $1^{\circ}$ e no $3^{\circ}$ trimestres e, também, quando a paciente é internada na maternidade em trabalho de parto ${ }^{32}$.

Apesar de tal recomendação, adotada pelo PNPH desde 2000, muitas mulheres não fazem mais de um exame VDRL durante todo o período gestacional ${ }^{19}$. Atrelado a isso está o fato das gestantes não saberem a importância dos exames, o que evidencia a inexistência de aconselhamento, o que consiste em uma situação comum nos serviços de saúde. Para orientá-las e subsidiar o aconselhamento, mostrase necessária a inserção de ações de vigilância epidemiológica, com o fornecimento de informações relevantes, confiáveis e atualizadas ${ }^{18}$.

As evidências epidemiológicas podem ser identificadas pelo índice de prevalência da sífilis gestacional. A OMS estabeleceu que, quando a

\section{As desigualdades sociais em saúde parecem apoiar a hipótese de que a sifilis gestacional se relaciona ao baixo nível socioeconômico...}


prevalência da IST se situa ao redor de $5 \%$ na população geral, ela representa um problema importante de saúde para a localidade ou o país em questã $0^{16}$. Os estudos encontrados nesta revisão foram conduzidos com gestantes com acesso prénatal, entretanto, os autores ${ }^{16}$ destacam que, se fossem acrescentadas as gestantes sem cobertura pré-natal, os demais individuos sexualmente ativos (de ambos os sexos) e os casos não notificados, muito provavelmente os números seriam alarmantes.

A maior prevalência de sífilis na gestação é encontrada em mulheres com menor escolaridade, sem assistência pré-natal e atendidas em serviços públicos. Ou seja, são estes os grupos populacionais mais expostos à infecção, que necessitam de maior atenção por parte dos programas de controle da sifilis gestacional ${ }^{33}$.

Entretanto, depreende-se que o controle da doença não seria necessário se houvesse estratégias de prevenção, seja da sífilis congênita ou da gestacional. Portanto, pondera-se que a condução de estudos que contribuam para o conhecimento e o entendimento da epidemiologia do agravo podem subsidiar um melhor planejamento das medidas de educação e de prevenção, principalmente nos grupos mais vulneráveis, promovendo ações para a redução da transmissão vertical da sífilis ${ }^{34-35}$.

Partindo dos programas e das políticas existentes na área da saúde da mulher, pode-se pensar em uma erradicação da sífilis se houver investimentos em atividades preventivas assistenciais. Sendo assim, há meios disponibilizados pelas três esferas de poder (federal, estadual e municipal) que podem propiciar a erradição desse problema de saúde pública, cabendo aos profissionais da saúde aumentar seus esforços a fim de tornar os serviços mais acessiveis, eficientes e organizados para atender a essa clientela ${ }^{22}$.

\section{CONCLUSÃO}

Os achados desta revisão evidenciaram lacunas na produção científica brasileira sobre estratégias de prevenção da sífilis gestacional, promovidas pelos profissionais da saúde nos diferentes cenários de atuação, principalmente no âmbito assistencial. Verificou-se, ainda, a importância da adesão ao tratamento e da inserção dos parceiros sexuais nesse seguimento, tendo em vista sua relevância para a prevenção das IST.

Considera-se necessária a ampliação da busca por estudos em outros países e da investigação de outros

\section{A maior prevalência de sífilis na gestação é encontrada em mulheres com menor escolaridade...}

achados sobre a produção científica na área da saúde sobre sífilis, o que constitui, assim, uma limitação deste estudo. Ao mesmo tempo, esta revisão permitiu apontar um caminho para o desenvolvimento de pesquisas e ações assistenciais voltadas à prevenção da sífilis, no período gestacional, que contribuam para a qualificação das ações adotadas no pré-natal.

\section{CONTRIBUIÇÃO DOS AUTORES}

Natália da Silva Gomes e Lisie Alende Prates contribuíram com a realização da pesquisa, o delineamento do estudo e a redação e revisão crítica do manuscrito. Rhayanna de Vargas Perez, Camila Xavier Fialho, Mari Luci Chalme da Silva e Pâmela da Rosa Gonzalez contribuíram com a redação do manuscrito.

\section{REFERÊNCIAS}

1. Avelleira JCR, Bottino G. Sífilis: diagnóstico, tratamento e controle. An Bras Dermatol [serial on the internet]. 2006 [cited 2018 Sep 15];81(2):11126. Available from: https://www.scielo.br/pdf/abd/ v81n2/v81n02a02.pdf

2. Brasil. Protocolo clínico e diretrizes terapêuticas para prevenção da transmissão vertical de HIV, sífilis e hepatites virais. Brasília (DF): Ministério da Saúde; 2015.

3. Brasil. Diretrizes para o controle da sífilis congênita. Brasília (DF): Ministério da Saúde; 2006.

4. Sistema de Informática do Sistema Único de Saúde [homepage on the internet]. Date unknown [cited 2020 Jan 2]. Available from: http://datasus. saude.gov.br

5. Cordeiro AM, Oliveira GM, Rentería JM, Guimarães CA. Revisão sistemática: uma revisão narrativa. Rev Col Bras Cir [serial on the internet]. 2007 [cited 2018 Sep 15];34(6):428-31. Available from: http:// www.scielo.br/pdf/rcbc/v34n6/11

6. Brasil. Portaria n. 569, de $1^{0}$ de junho de 2000. Institui o Programa de Humanização no Pré-Natal 
e Nascimento. Brasília (DF): Ministério da Saúde; 2000 .

7. Barbosa AJC, Bertin F, Silva RMM, Faller JW, Silva-Sobrinho RA, Zilly A. Perfil comportamental de gestantes atendidas no centro de testagem e aconselhamento em município de fronteira. Cogitare Enferm [serial on the internet]. 2016 [cited 2018 Sep 15];21(1):1-8. Available from: https://revistas. ufpr.br/cogitare/article/view/42957/27660

8. Costa MCO, Santos BC, Souza KEP, Cruz NLA, Santana MC, Nascimento OC. HIV/aids e sifilis entre gestantes adolescentes e adultas jovens: fatores de exposição e risco dos atendimentos de um programa de DST/HIV/aids na rede pública de saúde/SUS, Bahia, Brasil. Rev Baiana Saúde Pública [serial on the internet]. 2011 [cited 2018 Sep 15];35(Suppl 1):179-95. Available from: http://files.bvs.br/ upload/S/0100-0233/2011/v35nSupl1/a2308.pdf

9. Domingues RMSM, Hartz ZMA, Leal MC. Avaliação das ações de controle da sífilis e do HIV na assistência pré-natal da rede pública do Município do Rio de Janeiro, Brasil. Rev Bras Saúde Mater Infant [serial on the internet]. 2012 [cited 2018 Sep 15];12(3):26980. Available from: http://www.scielo.br/scielo. php? pid $=$ S1519-38292012000300007\&script $=$ sci abstract\&th $\mathrm{g}=\mathrm{pt}$

10. Andrade RFV, Lima NBG, Araújo MAL, Silva DMA, Melo SP. Conhecimento dos enfermeiros acerca do manejo da gestante com exame de VDRL reagente. DST $\mathrm{J}$ Bras Doenças Sex Transm [serial on the internet]. 2011 [cited 2018 Sep 15];23(4):188-93. Available from: http://www.dst.uff.br/revista23-4- 2011/8. Conhecimento $\% 20$ dos $\% 20$ Enfermeiros $\% 20$ acerca $\% 20$ do\%20Manejo.pdf

11. Araújo MAL, Silva DMA, Silva RM, Gonçalves MLC. Análise da qualidade dos registros nos prontuários de gestantes com exame de VDRL reagente. Rev APS [serial on the internet]. 2008 [cited 2018 Sep 15];11(1):4-9. Available from: https://periodicos. ufjf.br/index.php/aps/article/view/14172

12. Domingues RMSM, Saracen V, Hartz ZMA, Leal MC. Sífilis congênita: evento sentinela da qualidade da assistência pré-natal. Rev Saúde Pública [serial on the internet]. 2013 [cited 2018 Sep 15];47(1):147-57. Available from: http:// www.scielo.br/scielo.php?script $=$ sci arttext\&pid $=$ S0034-89102013000100019

13. Suto CSS, Silva DL, Almeida ES, Costa LEL, Evangelista TJ. Assistência pré-natal a gestante com diagnóstico de sífilis. Rev Enferm Atenção Saúde [serial on the internet]. 2016 [cited 2018 Sep 15];5(2):18-33. Available from: http://seer.uftm. edu.br/revistaeletronica/index.php/enfer/article/ view/1544
14. Inagaki $A D M$, Oliveira $L A R$, Oliveira MFB, Santos RCS, Araújo RM, Alves JAB, et al. Soroprevalência de anticorpos para toxoplasmose, rubéola, citomegalovírus, sifilis e HIV em gestantes sergipanas. Rev Soc Bras Med Trop [serial on the interne]. 2009 [cited Sep 15];42(5):532-6. Available from: http://www.scielo.br/scielo.php?pid=S0037$\underline{86822009000500010 \& s c r i p t=s c i \quad \text { abstract } \& t \operatorname{lng}=p t}$

15. Sá RAM, Bórnia RBG, Cunha AA, Oliveira CA, Rocha GPG, Giordano EB. Sífilis e gravidez: avaliação da prevalência e fatores de risco nas gestantes atendidas na maternidade de escola - UFRJ. DST J Bras Doenças Sex Transm [serial on the internet]. 2001 [cited 2018 Sep 15];13(4):6-8. Available from: http://www.dst.uff.br/revista13-4-2001/c1.pdf

16. Benzaken AS, García EG, Rodrigues A0, Mora C, Sánchez MS, Sardinha JCG. "Madre Fronteriza"; estudo conjunto de prevalência de sífilis tricomoníase e HIV em gestantes da tríplice fronteira do Alto Rio Solimões. DST J Bras Doenças Sex Transm [serial on the internet]. 2004 [cited 2018 Sep 15];16(3):15-23. Available from: http://cpa.sites.uff.br/wp-content/ uploads/sites/303/2018/01/r16-3-2004-2.pdf

17. Kupek E, Oliveira JF. Transmissão vertical do HIV, da sífilis e da hepatite B no município de maior incidência de AIDS no Brasil: um estudo populacional no período de 2002 a 2007. Rev Bras Epidemiol [serial on the internet]. 2012 [cited 2018 Sep 15];15(3):478-87. Available from: http://www. scielo.br/scielo.php?script $=$ sci arttext \& pid $=\$ 1415$ 790X2012000300004

18. Macêdo VC, Bezerra AFB, Frias PG, Andrade CLT. Avaliação das ações de prevenção da transmissão vertical do HIV e sifilis em maternidades públicas de quatro municípios do Nordeste brasileiro. Cad Saúde Pública [serial on the internet]. 2009 [cited 2018 Sep 15];25(8):1679-92. Available from: http://www.scielo.br/scielo.php?script=sci abstract\&pid=S0102-311X2009000800004\&lng=en\& $\underline{\mathrm{nrm}}=\mathrm{i}$ so \& $\mathrm{t} \operatorname{lng}=\mathrm{pt}$

19. Santos NP, Castro BG, Rios-Grassi MF. Aplicação do Protocolo do Projeto Nascer Maternidades em uma maternidade de referência em Feira de Santana, Bahia, Brasil. Rev Bras Saúde Mater Infant [serial on the internet]. 2009 [cited 2018 Sep 15];9(1):6976. Available from: http://www.scielo.br/scielo. php?pid=S1519-38292009000100008\&script=sci abstract\&tlng $=p t$

20. Nascimento MI, Cunha AA, Guimarães EV, Alvarez FS, Oliveira SRSM, Boas ELV. Gestações complicadas por sífilis materna e óbito fetal. Rev Bras Ginecol Obstet [serial on the internet]. 2012 [cited 2018 Sep 15];34(2):56-62. Available from: http://www.scielo. $\mathrm{br} / \mathrm{pdf} / \mathrm{rbgo} / \mathrm{v} 34 \mathrm{n} 2 / \mathrm{a} 03 \mathrm{v} 34 \mathrm{n} 2 . \mathrm{pdf}$

21. Domingues RMSM, Lauria LM, Saraceni V, Leal MC. Manejo da sífilis na gestação: conhecimentos, práticas e atitudes dos profissionais pré-natalistas 
da rede SUS do Município do Rio de Janeiro. Ciênc Saúde Colet [serial on the internet]. 2013 [cited 2018 Sep 15];18(5):1341-51. Available from: http:// www.scielo.br/pdf/csc/v18n5/19.pdf

22. Oliveira DR, Figueiredo MSN. Abordagem conceitual sobre a sífilis na gestação e o tratamento de parceiros sexuais. Enferm Foco (Brasília) [serial on the internet]. 2011 [cited 2018 Sep 15];2:108-11. Available from: http://revista.cofen.gov.br/index. php/enfermagem/article/view/106

23. Magalhães DMS, Kawaguchi IAL, Dias A, Calderon IMP. Sífilis materna e congênita: ainda um desafio. Cad Saúde Pública [serial on the internet]. 2013 [cited 2018 Sep 15];29(6):1109-20. Available from: http://www.scielo.br/scielo.php?script=sci arttext\&pid $=$ S0102-311X2013000600008

24. Reis HLB, Chambô Filho A, Sabino JB, Caldellas S. Sífilis recente em gestante e efeito prozona na sorologia: relato de caso. DST J Bras Doenças Sex Transm [serial on the internet]. 2007 [cited 2018 Sep 15];19(3-4):173-6. Available from: http://www. dst.uff.br/revista19-3-2007/10.pdf

25. Brandão JEC, Nina MPSN, Cervelli IK, Obrar AM, Terra AS, Sion F, et al. Soroprevalência de sífilis em gestantes HIV-negativas, obtida em três testes diagnósticos: VDRL, ELISA e TPHA. DST J Bras Doenças Sex Transm [serial on the internet]. 2002 [cited 2018 Sep 15];14(4):28-31. Available from: https:// pesquisa.bvs.br/ripsa/resource/pt/lil-329592

26. Grumach AS, Ferraroni NS. 0 papel da penicilina na medicina moderna. DST J Bras Doenças Sex Transm [serial on the internet]. 2006 [cited 2018 Sep 15];18(1):7-13. Available from: http://www.dst.uff. br/revista18-1-2006/2.pdf

27. Campos ALA, Araújo MAL, Melo SP, Andrade RFV, Gonçalves MLC. Sífilis em parturientes: aspectos relacionados ao parceiro sexual. Rev Bras Ginecol Obstet [serial on the internet]. 2012 [cited 2018 Sep 15];34(9):397-402. Available from: http:// www.scielo.br/scielo.php?script $=$ sci arttext\&pid =S0100-72032012000900002

28. Feliz MC, Medeiros ARP, Rossoni AM, Tahnus T, Pereira AMVB, Rodrigues C. Aderência ao seguimento no cuidado ao recém-nascido exposto à sífilis e características associadas à interrupção do acompanhamento. Rev Bras Epidemiol [serial on the internet]. 2016 [cited 2018 Sep 15];19(4):72739. Available from: http://www.scielo.br/scielo. php?pid =S1415-790X2016000400727\&script =sci abstract\&tlng $=p t$

29. Macêdo VC, Lira PIC, Frias PG, Romaguera LMD, Caires SFF, Ximenes RAA. Fatores de risco para sífilis em mulheres: estudo caso-controle. Cad Saúde Pública [serial on the internet]. 2017 [cited 2018 Sep 15];(51):78. Available from: http://www.scielo. br/scielo.php?script $=$ sci arttext\&pid=S0034-
$89102017000100268 \& \operatorname{lng}=$ en $\& n r m=i s o \& t \operatorname{lng}=p t$

30. Hebmuller MG, Fiori HH, Lago EG. Gestações subsequentes em mulheres que tiveram sífilis na gestação. Ciênc Saúde Colet [serial on the internet]. 2015 [cited 2018 Sep 15];20(9):2867-78. Available from: http://www.scielo.br/pdf/csc/v20n9/14138123-csc-20-09-2867.pdf

31. Organização Mundial da Saúde. Recomendações da OMS sobre cuidados pré-natais para uma experiência positiva na gravidez. Rio de Janeiro: 0MS; 2016.

32. Brasil. Atenção ao pré-natal de baixo risco. Brasília (DF): Ministério da Saúde; 2012.

33. Domingues RMSM, Szwarcwald CL, Souza Junior PRB, Leal MC. Prevalência de sífilis na gestação e testagem pré-natal: Estudo Nascer no Brasil. Rev Saúde Pública [document on the internet]. 2014 [cited 2018 Sep 15];48(5):766-74. Available from: http://www.scielo.br/pdf/rsp/v48n5/pt 0034-8910rsp-48-5-0766.pdf

34. Cavalcante PAM, Pereira RBL, Castro JGD. Sífilis gestacional e congênita em Palmas, Tocantins, 20072014. Epidemiol Serv Saúde [serial on the internet]. 2017 [cited 2018 Sep 15];26(2):255-64. Available from: http://www.scielo.br/scielo.php?pid=S2237$96222017000200255 \&$ script $=s c i$ abstract\&tlng $=p t$

35. Nunes JT, Marinho ACV, Davim RMB, Silva GG0, Feliz RS, Martino MMF. Sífilis na gestação: perspectivas e condutas do enfermeiro. Rev Enferm UFPE On Line [serial on the internet]. 2017 [cited 2018 Sep 15];11(12):4875-84. Available from: https:// periodicos.ufpe.br/revistas/revistaenfermagem/ article/download/23573/25297
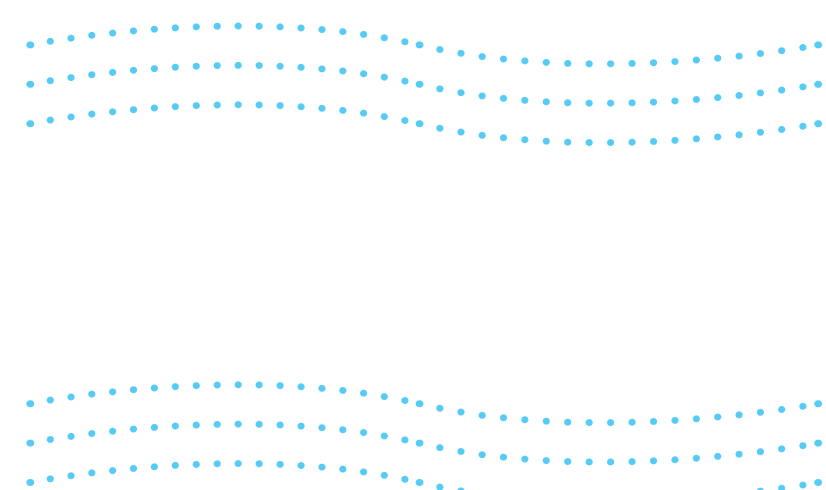